Research report

\title{
Temporal dynamics of Immediate Early Gene expression during cellular consolidation of spatial memory
}

\author{
Daniel N. Barry*, Sean Commins \\ Department of Psychology, Maynooth University, Co. Kildare, Ireland
}

\section{A R T I C L E I N F O}

\section{Article history:}

Received 14 December 2016

Received in revised form 4 March 2017

Accepted 9 March 2017

Available online 19 March 2017

\section{Keywords:}

Spatial navigation

Memory

Cellular consolidation

Immediate early genes

\begin{abstract}
A B S T R A C T
The consolidation of newly acquired memories on a cellular level is thought to take place in the first few hours following learning. This process is dependent on de novo protein synthesis during this time, which ultimately leads to long-term structural and functional neuronal changes and the stabilisation of a memory trace. Immediate early genes (IEGs) are rapidly expressed in neurons following learning, and previous research has suggested more than one wave of IEG expression facilitates consolidation in the hours following learning. We analysed the expression of Zif268, c-Fos and Arc protein in a number of brain regions involved in spatial learning either $90 \mathrm{~min}$, four hours or eight hours following training in the Morris water maze task. Consistent with the role of IEGs in the earliest stages of consolidation, a single wave of expression was observed in most brain regions at $90 \mathrm{~min}$, however a subsequent wave of expression was not observed at eight hours. In fact, Zif268 expression was observed to fall below the levels of naïve controls at this time-point in the medial prefrontal and perirhinal cortices. This may be indicative of synaptic downscaling in these regions in the hours following learning, and an important marker of the consolidation of spatial memory.
\end{abstract}

(c) 2017 Elsevier B.V. All rights reserved.

\section{Introduction}

Cellular consolidation refers to the initial stabilisation of a memory trace in the hours following learning [1]. Protein synthesis inhibitors administered during this time dirsupt memory formation, implicating de novo protein synthesis in this process [2]. These proteins are involved in the remodelling of synapses, resulting in a more enduring and stable memory [3]. The expression of Immediate Early Genes (IEGs) consitutes the cell's earliest genomic response to stimulation, and these proteins can either directly modify the structure and function of a cell to stabilise a memory, or activate late-response genes to facilitate cellular consolidation [4]. Synaptic plasticity in the hippocampus is disrupted in mice lacking these genes, with Zif268 Knockout (KO) mice showing impaired late-Long Term Potentiation (LTP) in the dentate gyrus, while lateLTP and Long Term Depression (LTD) is impaired in CA1 of Arc $\mathrm{KO}$ mice. A corresponding deficit in learning is also observed in these animals; Zif268 KO mice fail to reactivate the same place

\footnotetext{
* Corresponding author. Present address: Wellcome Trust Centre for Neuroimaging, Institute of Neurology, University College London, 12 Queen Square, London, WC1N3BG, UK.

E-mail addresses: daniel.barry@ucl.ac.uk (D.N. Barry), sean.commins@nuim.ie (S. Commins).
}

cell ensemble in CA1 $24 \mathrm{~h}$ after exploration of a novel environment [5]. The use of antisense oligodeoxynucleotides (AS-ODNs) which block the translation of mRNA into protein, has also revealed the necessity of IEG expression for memory formation. c-Fos antisenseODNs infused into CA3 before training in the radial arm maze has been shown to increase both working and reference memory errors [6]. Arc AS-ODNs administered into the hippocampus either before or immediately after training in the water maze task disrupts retention $48 \mathrm{~h}$ later without affecting acquisition, whereas administration eight hours after training had no effect [7], highlighting the time-sensitive role of these proteins in the early stages of consolidation.

However, there is some evidence that the role of IEG expression in consolidation is not just limited to the first few hours. During resting periods following spatial exploration, IEG expression is observed in the majority of CA1 neurons initially active during learning, suggesting a re-activation of the neural ensemble [8]. Upregulation of Arc and Zif268 is also observed in the cortex during REM sleep four hours after spatial exploration [9]. Ramirez-Amaya, Vazdarjanova [10] provided further evidence of multiple "waves" of consolidation, where at eight hours, but not four hours, following spatial exploration, CA1, CA3 and the parietal cortex showed elevated Arc expression, which was again observed in CA3 and the parietal cortex at $24 \mathrm{~h}$. Double labelling of Arc mRNA and protein 
confirmed that $81 \%$ of neurons activated at $8 \mathrm{~h}$, and $82 \%$ of neurons activated at $24 \mathrm{~h}$ belonged to the original ensemble active during exploration. However, while this multiple wave of IEG expression has been induced through electroconvulsive shock in mice, where a second wave of Arc expression has been observed in CA1 at eight hours, but not four or six hours following treatment [11], this phenomenon was not replicated under naturalistic conditions of spatial exploration. Furthermore, Arc, Zif268 and c-Fos mRNA expression in the hippocampus following place learning in the water maze drops to caged control levels or below at six hours [12], corresponding approximately to an eight hour time-point for IEG protein. Therefore the evidence for multiple waves of IEG expression over the course of consolidation of spatial memory remains inconclusive, and may differ according to the IEG used and region under investigation.

In order to resolve this ambiguity in the literature and conduct a more comprehensive investigation into temporal dynamics of IEGs in cellular consolidation, we examined the expression of Zif268, c-Fos and Arc protein in the rat hippocampus, medial prefrontal, entorhinal, perirhinal, retrosplenial, and parietal cortices, at $90 \mathrm{~min}$, four hours and eight hours after three days of training in the reference memory version of the water maze.

\section{Materials and methods}

\subsection{Subjects}

Twenty-six male Wistar rats, obtained from Charles River Laboratories, UK, were used as subjects in this experiment. Subjects were approximately three months old and weighed 200-300 g at the beginning of experimentation. All animals were housed three per cage in plastic-bottomed cages $(56 \mathrm{~cm} \times 38 \mathrm{~cm}$ wide and $22 \mathrm{~cm}$ high; NKP Cagers, UK) with a $3 \mathrm{~cm}$ layer of woodchip bedding, paper strip nesting material and cardboard tubes for environmental enrichment. Animals were housed in a conventional facility in a temperature controlled environment $\left(21 \pm 1^{\circ} \mathrm{C}\right)$, which was maintained on a fixed 12:12 h light-dark cycle (07:00-19:00). All rats were given ad libitum access to water and food pellets (Red Mills, Ireland). Experimentation took place during the light phase and all subjects were well handled before experimentation began. Guidelines for the maintenance and experimentation of animals conformed to the Department of Health and Children (Ireland) guidelines under statutory instrument (S.I.) No. 543 of 2012 and the European directive 2010/63/EU. All experiments were also approved by Maynooth University Ethics board.

\subsection{Spatial task}

The Morris water maze [13], was chosen as the spatial task as it is an extensively studied and particularly demanding task of allocentric spatial memory. The water maze was made of black fibreglass $1.7 \mathrm{~m}$ in diameter and $36 \mathrm{~cm}$ in depth, mounted on a platform $70 \mathrm{~cm}$ above the floor [14]. The maze was filled with water to a depth of $20 \mathrm{~cm}$ and maintained at a temperature of $20 \pm 1^{\circ} \mathrm{C}$. The escape platform was $13.5 \mathrm{~cm}$ in width and $18 \mathrm{~cm}$ in height, and was placed in the centre of the northeast quadrant of the maze, submerged $2 \mathrm{~cm}$ below the surface of the water. The water maze was surrounded by a black curtain which obscured the rest of the room from view. Three distal cues were available, two $25 \mathrm{w}$ bulbs which were suspended from the ceiling at a distance of $75 \mathrm{~cm}$ from the edge of the pool and at an angle of $60^{\circ}$, one in the north-east and the other in the south-east, and a rectangular piece of white card $(55 \mathrm{~cm} \times 81 \mathrm{~cm})$ which was also suspended from the ceiling on the west side of the maze. The animal's movements for each trial were recorded by a camera positioned directly above the centre of the maze. This information was collected by the digital tracking software EthoVision (Noldus Information Technologies, Wageningen, Netherlands).

Rats were randomly allocated to one of three experimental groups; $90 \mathrm{~min}$, four hours, or eight hours post-training ( $n=7$ per group). Animals were trained for three consecutive days in the water maze, with four trials per day (see Harvey, McGauran [15]). Animals were placed into the water maze from one of four starting positions, either north, south, east or west, with each starting position used just once per day. Animals were allowed $60 \mathrm{~s}$ to locate the escape platform, after which they would be guided to the platform by the experimenter, and allowed to remain there for $15 \mathrm{~s}$. Following an inter-trial interval of $10 \mathrm{~s}$, the animal was placed back into the maze to begin the next trial. Following training every day, and prior to perfusion on the last day of training, rats were returned to their home cages. A naïve control group $(n=5)$ which was not exposed to the water maze apparatus was included to provide a baseline measure of IEG expression.

\subsection{Immunohistochemistry}

Immunohistochemical protocol for the detection of Zif268, cFos and Arc protein was carried out on all groups of animals (see also Barry, Coogan [16]). Depending on the experimental group, either $90 \mathrm{~min}$, four hours or eight hours after the final training trial on day three, rats were deeply anaesthetised with an intraperitoneal injection of sodium pentobarbital $(100 \mathrm{mg} / \mathrm{kg}$, Euthatal), and subsequently perfused transcardially with ice cold $0.9 \%$ phosphate buffered saline (PBS, Ph7.4), followed by $4 \%$ paraformaldehyde in $0.1 \mathrm{M}$ phosphate buffer (PB, Ph7.4). Brains were then rapidly removed and post-fixed in $4 \%$ paraformaldehyde overnight, and then transferred to a $30 \%$ sucrose solution and stored at $4{ }^{\circ} \mathrm{C}$. Coronal sections were cut at $40 \mu \mathrm{m}$ using a freezing microtome, with every fourth section taken for subsequent immunohistochemical analysis. Rats in the caged control group were taken directly from their home cages and sacrificed.

Prior to the immunohistochemical procedure, free floating sections were stored in $0.1 \mathrm{M}$ PB containing $0.01 \%$ sodium azide at $4{ }^{\circ} \mathrm{C}$. In order to minimise variation due to the immunohistochemical procedure, brain sections representing a particular region from all subjects were processed in a single batch. Sections were given two 10 min washes in $0.1 \mathrm{M} \mathrm{PB}$, followed by a $10 \mathrm{~min}$ wash in $0.1 \mathrm{M}$ PB containing $0.2 \%$ Triton-X-100 (PBX). A 20 min wash in $0.1 \mathrm{M}$ PB containing $1.5 \%$ hydrogen peroxide was then carried out. This was followed by another two washes in $0.1 \mathrm{M} \mathrm{PB}$ and one in PBX. Sections were then blocked for $60 \mathrm{~min}$ at room temperature in $5 \%$ normal goat serum (NGS) in $0.1 \mathrm{M} \mathrm{PBX}$. Sections were then incubated overnight in a primary antibody solution (2\% NGS in 0.1 M PBX). Labelling of Zif268, c-Fos and Arc were performed using the following primary antibodies: Zif268/Egr-1, rabbit polyclonal antibody raised against the $C$ terminus of human Egr-1 (dilution 1:3000; Santa Cruz Biotechnology); c-Fos, rabbit polyclonal antibody raised against the amino terminus of human c-Fos (dilution 1:2000; Santa Cruz Biotechnology); Arc, rabbit polyclonal antibody corresponding to amino acids 1-300 of Arc of human origin (dilution 1:800; Santa Cruz Biotechnology). After incubation with the primary antibody, sections were washed twice in $0.1 \mathrm{M}$ PB and once in PBX and then incubated with biotinylated secondary antibody (goat anti-rabbit, Jackson Laboratories, dilution $1: 400$ ) for $70 \mathrm{~min}$. Sections were again washed twice in $0.1 \mathrm{M} \mathrm{PB}$ and once in $0.1 \mathrm{M}$ PBX before incubation with avidin-biotin-peroxidase complex (0.4\%; Vector Laboratories) for $90 \mathrm{~min}$ in lightproof conditions at room temperature. Sections were then given two $10 \mathrm{~min}$ washes in PB followed by one 10 min wash in $0.1 \mathrm{M}$ sodium acetate, pH6. This was followed by visualisation of the antigen using the nickel DAB method with glucose oxidase (Sigma, Poole, UK) as the 
catalyst. Sections were reacted for standardised lengths of time to ensure similar staining intensity across experimental groups. Sections were then mounted onto gelatine-coated slides, dried, dehydrated, cleared in Histoclear (National Diagnostics, Hull, UK), and coverslipped using Eukitt (Sigma, Poole, UK).

\subsection{Regions of interest}

Based on the findings of lesion and imaging studies, a number of regions were chosen for analysis due to their likely involvement in spatial memory processes. These included the dorsal hippocampus (CA1, CA3, dentate gyrus), parahippocampal areas (lateral and medial entorhinal, and perirhinal cortices), the medial prefrontal cortex (anterior cingulate, prelimbic and infralimbic cortices), and the retrosplenial and parietal cortices.

\subsection{Image analysis}

Counts of IEG expressing cells were calculated using an automated computerised cell counting procedure, which eliminated bias which could arise from manual counting procedures. Images were taken of the 11 regions sampled using an Olympus DP12 digital camera, mounted on an Olympus BX51 microscope and captured using a $4 \times$ magnification. As this level of magnification usually sampled a larger area than that under investigation, in order to obscure adjacent brain regions during image acquisition, appropriately scaled images of the coronal sections in question, adapted from Paxinos and Watson (2007), were printed onto clear acetate, with all regions except those of interest blacked out, and these were positioned over the brain section during image acquisition. This approach allowed for maximal coverage of the area of interest (see also Barry, Coogan [16]). This procedure was carried out for all regions sampled except the hippocampus, where images were manually cropped following image acquisition. Numbers of immunopositive cells were analysed using the public domain program ImageJ (National Institute of Health, USA). Cell counts above a pre-defined brightness intensity threshold, and within a predefined particle area size were calculated. Counts from consecutive sections were averaged to produce a mean for each animal. The mean number of sections analysed per region, collapsed across all four groups and IEG analyses were as follows: CA1: 5.8 (SD 0.3), CA3: 5.8 (SD 0.3), DG: 5.8 (SD 0.3), lateral entorhinal cortex: 3.8 (SD 0.3 ), medial entorhinal cortex: 3.8 (SD 0.3), perirhinal cortex: 5.7 (SD 0.5), anterior cingulate cortex: 3.6 (SD 0.4), prelimbic cortex: 3.8(SD 0.3), infralimbic cortex: 3.8 (SD 0.3), retrosplenial cortex: 5.6 (SD 0.8), parietal cortex: 5.7 (SD 0.7). As all animals were processed as a single batch during the immunohistochemical procedure, a normalisation procedure was not necessary and raw values were used for statistical analyses [17].

\subsection{Statistical analysis}

All statistical analyses were carried out using SPSS (Version 20). Acquisition of the water maze across all groups was evaluated using a one-way repeated measures ANOVA with Bonferroni correction. Analyses of differences in latencies between groups were assessed using a mixed design ANOVA with day and group as the within and between group factors respectively. The significance of differences in IEG expression between groups was assessed using one-way ANOVA with Tukey post-hoc tests.

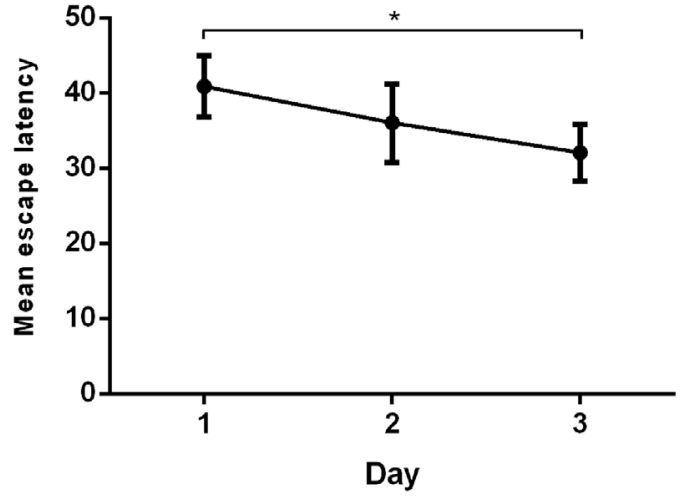

Fig. 1. Average daily escape latency in seconds ( \pm SEM) for all animals over three days of training in the Morris water maze.

\section{Results}

\subsection{Morris water maze acquisition}

An overall significant effect was found in time taken to escape the maze across the three days of training by all animals (one-way repeated measures ANOVA, $\left.F_{(2,40)}=3.84, p=0.030\right)$, with escape latencies lower on day three compared to day $1(p=0.041$, see Fig. 1). No difference in water maze escape latencies was observed across the $90 \mathrm{~min}$, four hour or eight hour groups, (mixed ANOVA, $\left.F_{(2,18)}=0.29, p>0.05\right)$ indicating performance across the three groups was comparable.

\subsection{IEG analysis}

\subsubsection{Zif 268}

A significant effect was found between groups in the anterior cingulate cortex (one-way ANOVA, $F_{(3,21)}=21.69, p<0.001$ ), with post-hoc Tukey tests revealing counts of Zif268 significantly increased $90 \mathrm{~min}$ following training compared to four hours $(p=0.003)$, eight hours $(p<0.001)$ and caged controls $(p<0.001)$. Counts were also significantly lower at eight hours than four hours $(\mathrm{p}=0.003)$ and lower than caged controls $(p=0.035)$. A significant difference was also found for the prelimbic cortex, $F(3,21)=32.27$, $p<0.001$, where counts of Zif268 decreased from 90 min posttraining to four hours $(p<0.001)$, eight hours $(p<0.001)$, and caged controls $(p<0.001)$. Zif268 counts were also significantly lower in the eight hour group than the four hour $(p=0.037)$ and caged control groups $(p=0.046$. Counts of Zif268 also differed across groups in the infralimbic cortex (one-way ANOVA, $F(3,22)=25.05$, $p<0.001$ ), with higher levels of Zif268 at 90 min post-acquisition than four hours $(p<0.001)$, eight hours $(p<0.001)$ and caged controls $(p<0.001)$. The initial increase in Zif268 at $90 \mathrm{~min}$ for all three regions had returned to baseline levels at four hours, with no difference from controls at this time point (all $p>0.05$, see Fig. $2 a$ ).

A significant difference was found across the three consolidation time-periods in CA1 (one-way ANOVA, $F_{(3,22)}=11.07, p<0.001$ ), with Zif268 counts significantly higher at $90 \mathrm{~min}$ than four hours $(p=0.027)$ and eight hours post-acquisition $(p<0.001)$. An effect for group was also found in CA3 (one-way ANOVA, $F_{(3,22)}=17.43$, $p<0.001$ ), with Zif-positive cell counts higher in the 90 min group than the four hour $(p=0.005)$, eight hour $(p<0.001)$, and caged control group $(p<0.001)$. No effect for group was found in the dentate gyrus (one-way ANOVA, $F_{(3,22)}=2.44, p>0.05$ ). Zif268 levels in CA1 and CA3 were no longer higher than caged controls at four hours (both $p>0.05$, see Fig. 2B).

A main effect for group was found in the lateral entorhinal cortex (one-way ANOVA, $F_{(3,21)}=12.74, p<0.001$ ), with counts sig- 
A

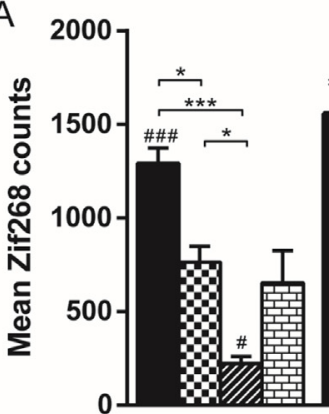

ACC

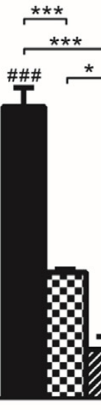

$\mathrm{PL}$

mPFC

C

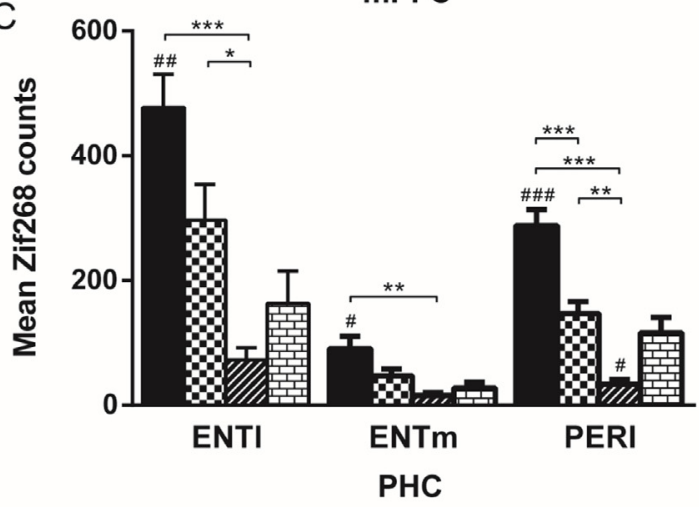

B

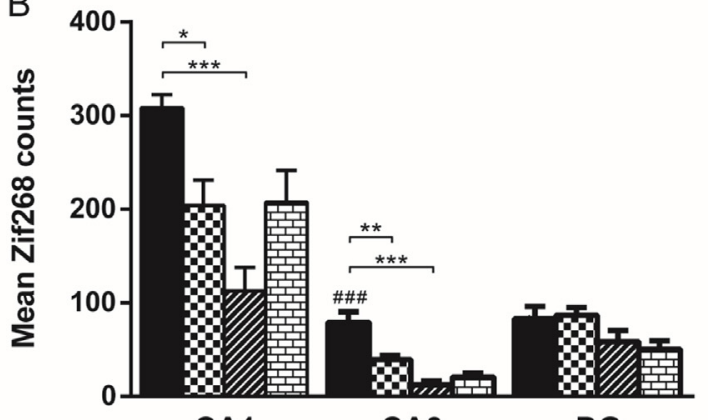

CA1

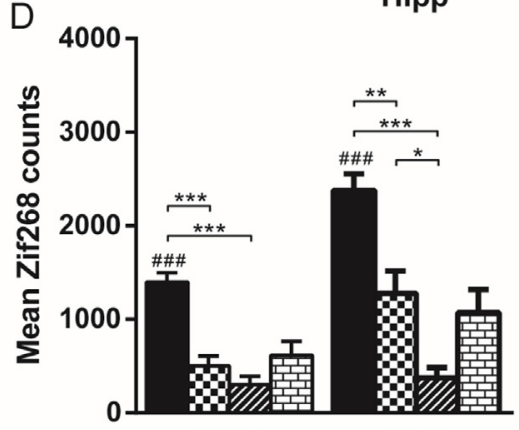

RSP
DG

\section{Minutes \\ (B) 4 Hours \\ WII 8 Hours \\ Controls}

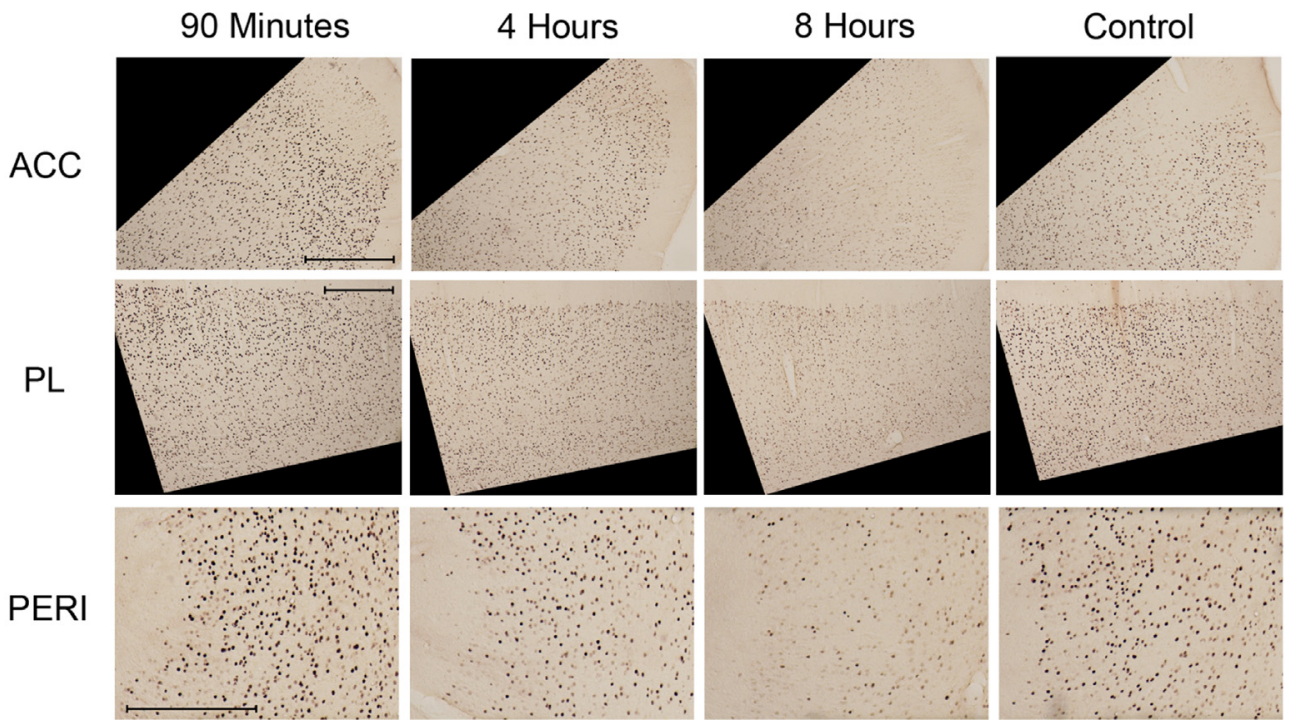

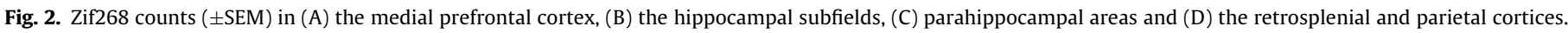

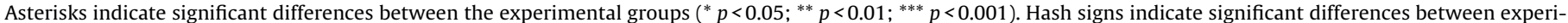
mental groups and caged controls (\# $p<0.05$; \#\# $p<0.01$; \#\#\# $p<0.001$ ).

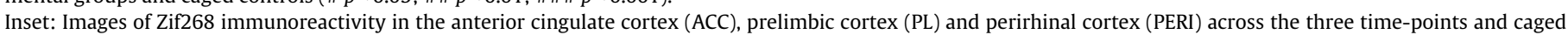
controls. Scale bar $=500 \mu \mathrm{m}$.

nificantly higher in the 90 min group than at 8 hours $(p<0.001)$ or the caged control group $(p=0.002)$, with counts also lower at eight hours than four hours $(p=0.02)$. An overall effect for group was found in the medial entorhinal cortex (one-way ANOVA, $\left.F_{(3,22)}=6.64, p=0.002\right)$, with Zif268 counts higher in the 90 min than the eight hour $(p=0.002)$ and caged control $(p=0.019)$ groups. A significant effect across groups was also found in the perirhinal cortex (one-way ANOVA, $F_{(3,22)}=30.36, p<0.001$ ), with a significant decrease in Zif268 counts from $90 \mathrm{~min}$ to four hours $(p<0.001)$ and eight hours following training $(p<0.001)$, as well as being significantly increased from baseline $(p<0.001)$. Counts at eight hours post-training were also significantly lower than four hours $(p=0.002)$ and caged controls $(p=0.049)$. Expression of Zif268 in the entorhinal and peririnal cortices did not differ from baseline by four hours post training (all $p>0.05$, see Fig. 2C).

A significant effect between groups was found in the retrosplenial cortex (one-way ANOVA, $F_{(3,21)}=21.50, p<0.001$ ), with Zif268 counts significantly higher at the 90 min than four hour $(p<0.001)$ 
and eight hour $(p<0.001)$ time points, as well as caged controls $(p<0.001)$. A difference across groups was also found in the parietal cortex (one-way ANOVA, $F_{(3,22)}=19.52, p<0.001$ ), with Zif268 counts significantly increased at 90 min following training compared to four hours $(p=0.002)$, eight hours $(p<0.001)$ and caged controls $(p<0.001)$. Counts were also significantly higher at four hours than eight hours $(p=0.013)$. Levels of Zif268 had returned to baseline by four hours in both regions ( $p>0.05$, see Fig. 2D).

\subsection{2. $\operatorname{Arc}$}

The expression of Arc was also assessed across the three time points and caged controls. A significant effect was found between groups in the anterior cingulate cortex (one-way ANOVA, $F_{(3,21)}=5.04, p=0.009$ ), with counts of Arc significantly increased 90 min following training compared to four hours $(p=0.020)$ and eight hours $(p=0.014)$. A significant difference was also found for the prelimbic cortex (one-way ANOVA, $F_{(3,20)}=5.40, p=0.007$ ), with counts of Arc decreased from $90 \mathrm{~min}$ to four hours $(p=0.018)$ and eight hours $(p=0.013)$. Counts of Arc also differed across groups in the infralimbic cortex (one-way ANOVA, $F_{(3,21)}=5.92$, $p=0.004)$, where levels of Arc were higher at $90 \mathrm{~min}$ than four hours $(p=0.011)$, eight hours $(p<0.008)$ and caged controls $(p=0.046)$. While increases in Arc expression from caged control levels approached significance in the anterior cingulate $(p=0.084)$ and prelimbic cortices $(p=0.057)$ at $90 \mathrm{~min}$, no medial prefrontal regions were higher than baseline levels at four or eight hours (all $p>0.05$, see Fig. $3 A$ ).

A significant difference in Arc expression was not found across groups in CA3 (one-way ANOVA, $F_{(3,21)}=2.75, p>0.05$ ), or the dentate gyrus (one-way ANOVA, $F_{(3,22)}=1.60, p>0.05$, see Fig. 3B). Arc staining was absent in area CA1.

The lateral entorhinal cortex showed a difference across groups (one-way ANOVA, $F_{(3,21)}=7.55, p=0.001$ ), with Arc expression significantly higher at $90 \mathrm{~min}$ post-training than four hours $(p=0.007)$, eight hours $(p=0.002)$ and caged controls $(p=0.024)$. A difference across groups was also found in the medial entorhinal cortex (oneway ANOVA, $\left.F_{(3,22)}=7.71, p=0.001\right)$, where Arc counts were higher at 90 min following training than four hours $(p=0.004)$, eight hours $(p=0.001)$ and caged controls $(p=0.045)$. A significant effect for group was also found in the perirhinal cortex (one-way ANOVA, $\left.F_{(3,21)}=9.65, p<0.001\right)$, where a significant decrease in Arc counts was observed from 90 min post-training to four hours $(p=0.002)$ and eight hours $(p<0.001)$, as well as being significantly increased from baseline $(p=0.004)$. Arc counts in the entorhinal and perirhinal cortices did not differ from caged controls at four or eight hours post training (all $p>0.05$, see Fig. $3 \mathrm{C}$ ).

A significant effect between groups was found in the retrosplenial cortex (one-way ANOVA, $F_{(3,21)}=5.82, p=0.005$ ), with Arc counts significantly higher at the $90 \mathrm{~min}$ time-point than four hours $(p=0.010)$ and eight hours $(p=0.007)$. A difference across groups was also found in the parietal cortex (one-way ANOVA, $F$ $(3,22)=5.80, p=0.004)$, with Arc counts significantly increased at 90 min after training compared to four hours $(p=0.008)$ and eight hours $(p=0.008)$. Arc counts did not differ significantly from caged controls in the retrosplenial cortex at any timepoint (all $p>0.05$ ). Arc counts in the parietal cortex approached a significant increase from baseline at $90 \mathrm{~min}(p=0.062)$, but were not significantly different from caged controls at four or eight hours ( $p>0.05$ see Fig. 3C)).

\subsection{3. c-Fos}

Analysis of c-Fos expression revealed a significant effect was found between groups in the anterior cingulate cortex (one-way ANOVA, $F_{(3,21)}=13.77, p<0.001$ ), with Tukey post-hoc tests revealing counts of c-Fos significantly increased 90 min following training compared to four hours $(p<0.001)$, eight hours $(p<0.001)$ and caged controls $(p=0.003)$. A significant difference was also found for the prelimbic cortex (one-way ANOVA, $F_{(3,20)}=12.62, p<0.001$ ), where counts of c-Fos decreased from $90 \mathrm{~min}$ post-acquisition to four hours $(p<0.001)$, eight hours $(p<0.001)$, and caged controls $(p=0.006)$. Counts of $c$-Fos also differed across groups in the infralimbic cortex (one-way ANOVA, $F_{(3,21)}=11.89, p<0.001$ ), with higher levels of c-Fos 90 min after training than four hours $(p<0.001)$, eight hours $(p<0.001)$ and caged controls $(p=0.004)$. cFos counts in all three medial prefrontal regions had returned to baseline levels at four and eight hours ( $p>0.05$, see Fig. 4A)

A significant difference was found across groups in CA1 (oneway ANOVA, $\left.F_{(3,21)}=4.36, p=0.016\right)$, where c-Fos counts were significantly higher at $90 \mathrm{~min}$ than eight hours post-training $(p=0.020)$. A significant difference across the three groups was also found in CA3 (one-way ANOVA, $F_{(3,21)}=4.21, p<0.018$ ), with higher $c$-Fos expression at 90 min following training than eight hours $(p=0.016)$. A main effect for group was found in the dentate gyrus (one-way ANOVA, $F_{(3,20)}=4.03, p=0.022$ ), with c-Fos counts higher at $90 \mathrm{~min}$ than eight hours $(p=0.019)$. Counts in CA1 $(p=0.054)$ and CA3 $(p=0.095)$ at the $90 \mathrm{~min}$ time point approached a significant increase from baseline, but did not differ from baseline at four or eight hours (all $p>0.05$ see Fig. 4B).

A difference across groups was also found in the lateral entorhinal cortex (one-way ANOVA, $F_{(3,22)}=8.78, p<0.001$ ), where c-Fos expression was significantly higher $90 \mathrm{~min}$ following training than four hours $(p=0.01)$, eight hours $(p<0.001)$ and caged controls $(p=0.018)$. A difference across groups was also found in the medial entorhinal cortex (one-way ANOVA, $F_{(3,21)}=6.24, p=0.003$ ), with higher c-Fos counts at the $90 \mathrm{~min}$ time-point than eight hours $(p=0.002)$. Significant differences were found in the perirhinal cortex (one-way ANOVA, $F_{(3,21)}=14.55, p<0.001$ ), where c-Fos expression was significantly higher at 90 min than four $(p<0.001)$ and eight hours post-acquisition $(p<0.001)$ as well as caged controls $(p=0.002)$. c-Fos expression in the lateral entorhinal and perirhinal cortex had returned to the levels of caged controls at four and eight hours (both $p>0.05$ ). Counts in the medial entorhinal cortex did not differ significantly from baseline at any timepoint but approached significance at $90 \mathrm{~min}$ post-training $(p=0.087$, see Fig. 4C).

A significant difference was found between the groups in the retrosplenial cortex (one-way ANOVA, $F_{(3,21)}=11.04, p<0.001$ ) with significantly higher c-Fos expression at 90 min post-training than four hours $(p<0.001)$, eight hours $(p<0.001)$ and caged controls $(p=0.006)$. A difference across groups was also found in the parietal cortex (one-way ANOVA, $F_{(3,20)}=5.45, p=0.007$ ), where c-Fos expression was significantly higher at the $90 \mathrm{~min}$ time-point than 4 hours $(p=0.012)$ and eight hours $(p=0.011)$. c-Fos counts in the retrosplenial cortex were not significantly different from baseline at four or eight hours $(p>0.05)$. c-Fos expression in the parietal cortex approached a significant increase from caged controls at $90 \mathrm{~min}$ post-training $(p=0.059)$, but not at later time points $(p>0.05$, see Fig. 4D).

\section{Discussion}

This experiment examined the changes in IEG expression in 11 brain regions at three separate time-points in the hours following learning in the Morris water maze in order to resolve the inconsistency in the literature regarding multiple waves of IEGfacilitated consolidation in the hours following spatial learning. The most notable finding was the apparent absence of a second wave of IEG expression at eight hours in any of the brain regions studied. In some regions the complete opposite was observed, with IEG expression significantly below basal levels at this time-point.

Zif268 expression was higher at $90 \mathrm{~min}$ following training than at eight hours or caged controls in CA1, CA3, the lateral and medial 
A

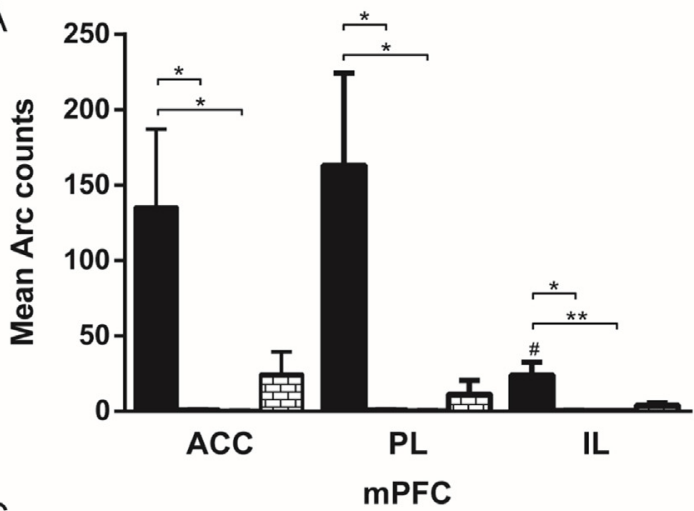

C

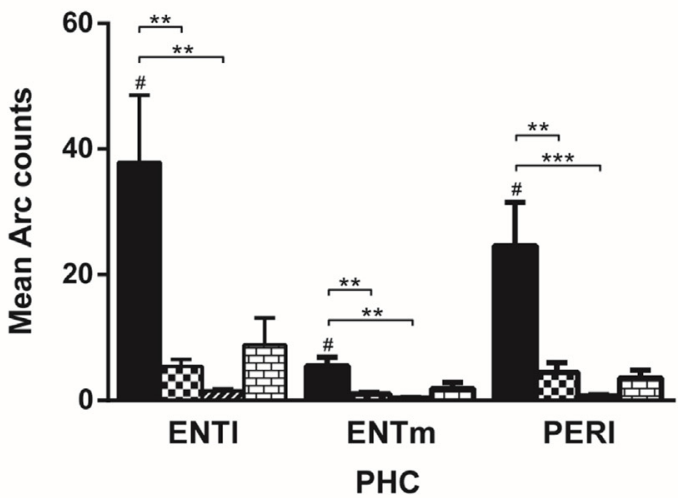

B

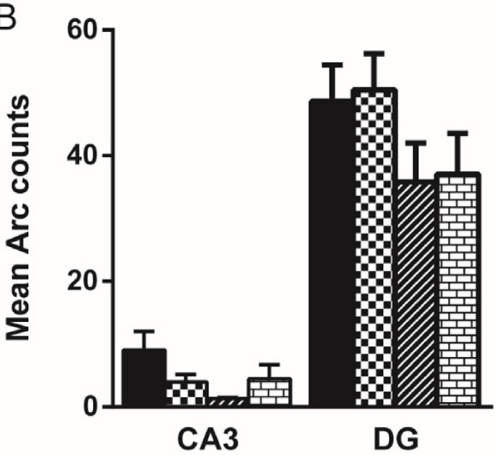

HIPP

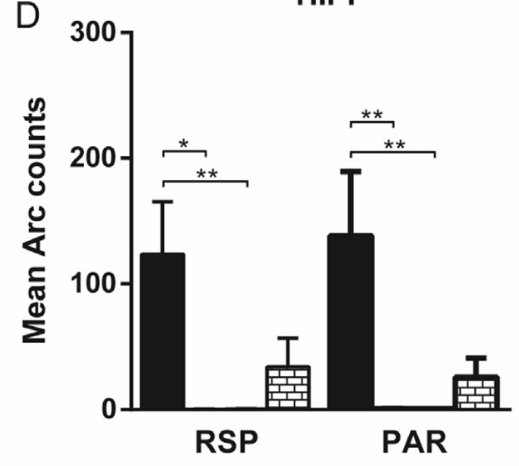

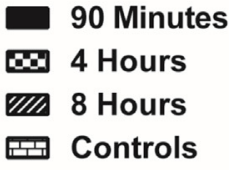

90 Minutes

WII 8 Hours

Controls

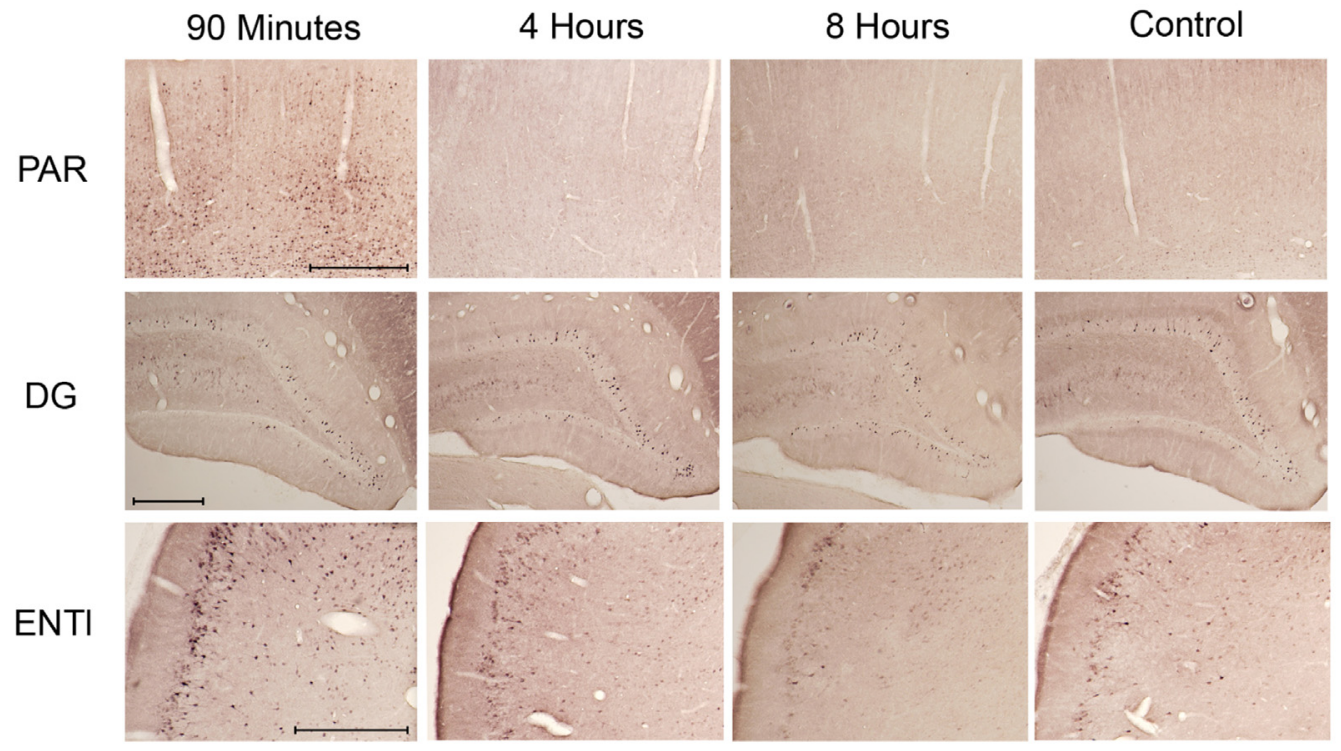

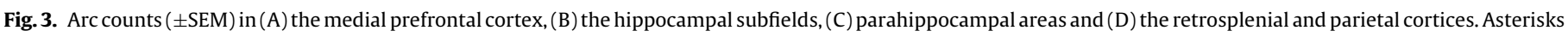

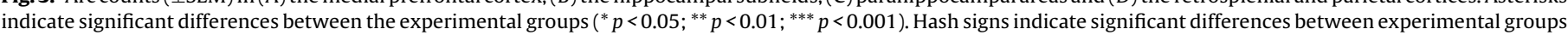
and caged controls (\# $p<0.05$ ).

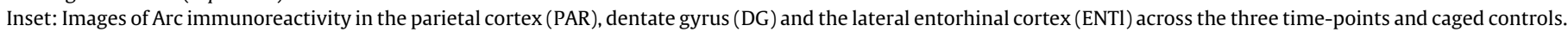
Scale bar $=500 \mu \mathrm{m}$.

entorhinal cortices, the retrosplenial, perirhinal and parietal cortices, and all three subregions of the medial prefrontal cortex. This is consistent with the time course of expression of this IEG, which is maximally expressed $90 \mathrm{~min}$ following experience [18], and has been shown to be upregulated at this time point in these areas following water maze training $[12,19,20]$. However in contrast to the findings of Ramirez-Amaya, Vazdarjanova [10], a "second wave" of IEG expression was not observed at eight hours post-training in any of the regions studied. This is surprising, as the spatial exploration of a novel environment in that study would not be nearly as taxing as the effortful learning required to perform the water maze task successfully. In fact, Zif268 expression at the eight hour time-point was even lower than caged controls in the perirhinal, anterior cingulate and prelimbic cortices. This depletion of IEG protein below caged control levels is in the opposite direction of the "multiple waves" of neural activity hypothesis and is an intriguing finding 
A
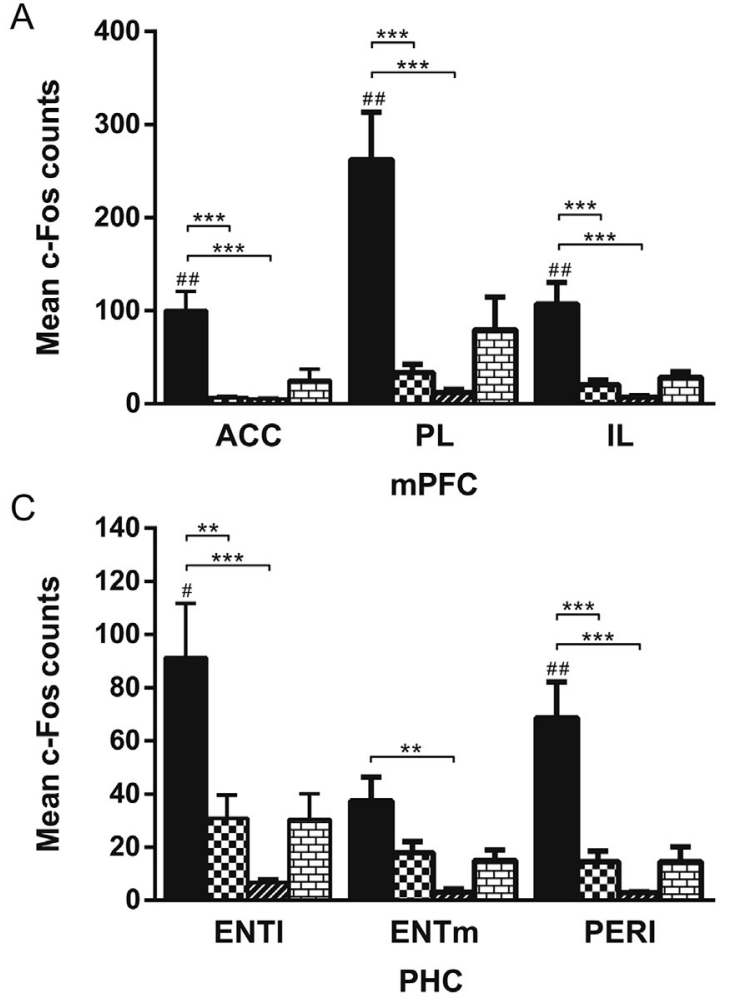

B

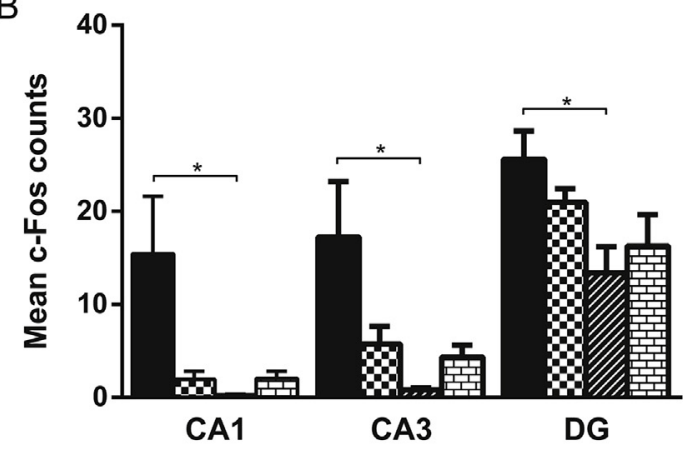

$\mathrm{D}$

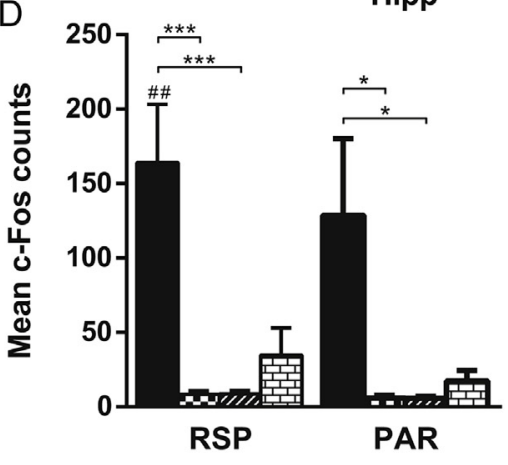

- 90 Minutes

DI 4 Hours

III 8 Hours

Controls

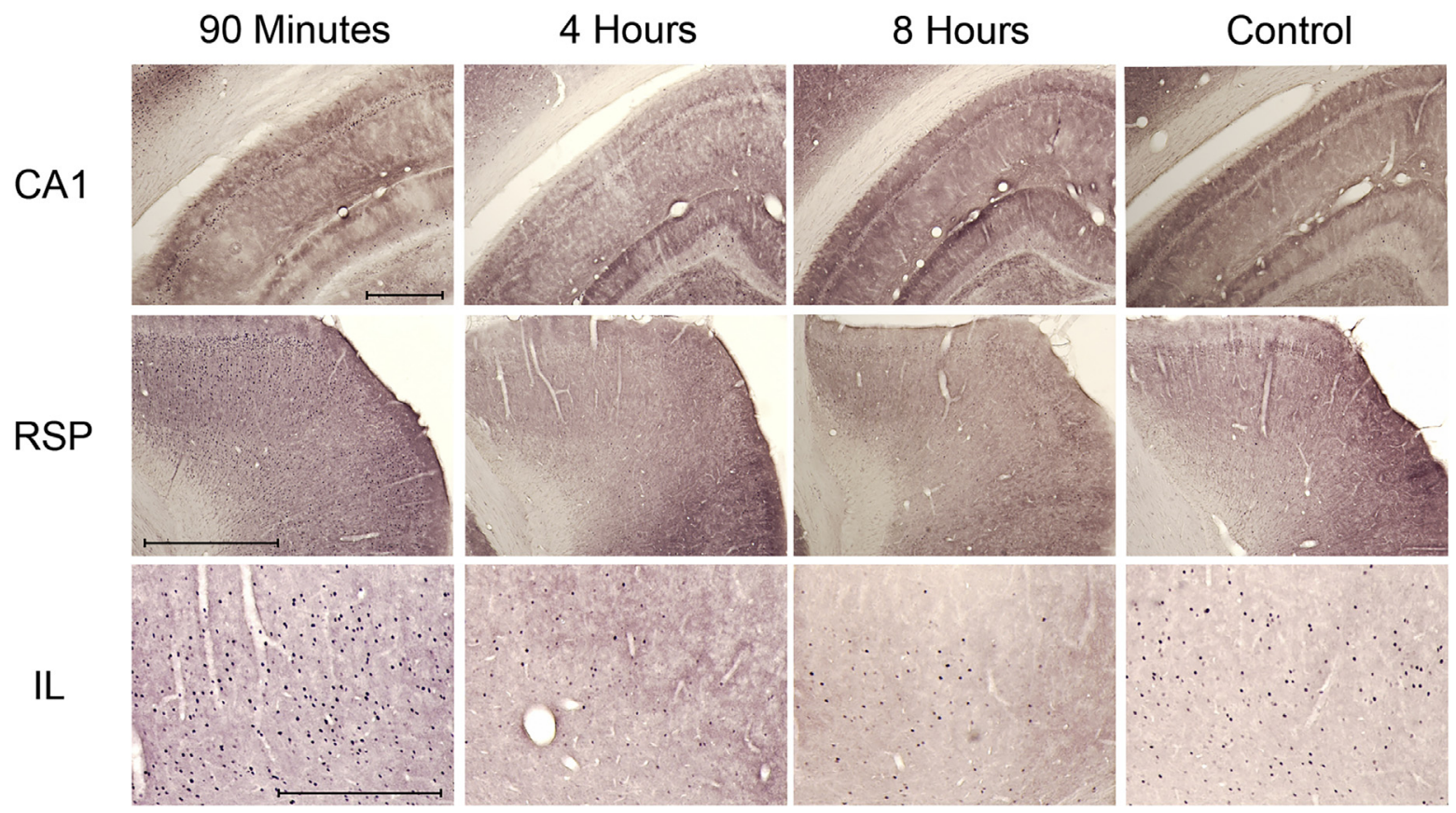

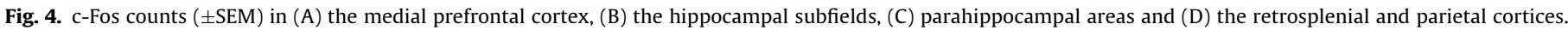

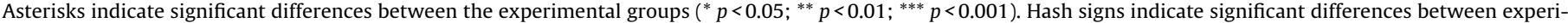
mental groups and caged controls (\# $p<0.05$; \#\# $p<0.01$ ).

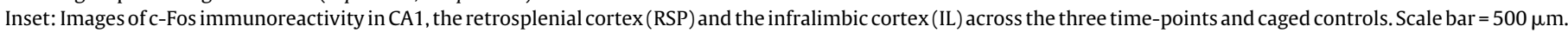

which warrants explanation. This phenomenon has been observed before by Guzowski, Setlow [12], where c-Fos mRNA in the hippocampus dropped below caged control levels six hours following training in the water maze. Furthermore, examining the timecourse of Zif268 expression following fear conditioning, Lonergan, Gafford [21] observed hippocampal expression to drop below caged control levels from 1.5 to $24 \mathrm{~h}$. Such marked downregulation in a wide range of genes involved in memory consolidation has also been observed $12 \mathrm{~h}$ following passive avoidance learning in rats [22]. The reduced IEG activity observed in the current study at the eight hour time-point could be atrributabe to synaptic downscaling, where neural activity is inhibited following Long-Term Potentiation to preserve network stability [23]. Therefore muted IEG expression at this time point may be a strong marker of mem- 
ory consolidation in the anterior cingulate, prelimbic and perirhinal cortices. One finding of Ramirez-Amaya, Vazdarjanova [10] which we have replicated here, is the prolonged IEG expression in the dentate gyrus from $90 \mathrm{~min}$ to eight hours. Whether this is the result of single or multiple waves of expression is unclear, and the failure to detect a significant increase from caged controls at any of the time-points makes this result difficult to interpret

Dramatic decreases in Arc expression from $90 \mathrm{~min}$ to four hours were observed in the lateral and medial entorhinal cortices, the retrosplenial, perirhinal and parietal cortices, and the anterior cingulate, prelimbic and infralimbic cortices, however a "second wave" of activity at eight hours was not observed. These findings, for the most part, are at variance with Ramirez-Amaya, Vazdarjanova [10] who used Arc as a marker of consolidation and found multiple waves of consolidation in CA3 and the parietal cortex, and support the findings of Penke, Chagneau [11], where only a single wave of expression was found following spatial exploration. IEG expression in the dentate gyrus appears to be largely unchanged over the eight hours although the non-significant increase from caged controls means these results should be interpreted with a degree of caution.

The analysis of c-Fos expression over the course of hours following spatial training also showed a similar initial increase at the 90 min time-point, with all regions displaying significantly higher levels of c-Fos at this stage when compared to eight hours or caged controls. The only regions where expression at four hours did not differ significantly from 90 min, were CA1, CA3, the dentate gyrus and the medial entorhinal cortex. Again however, similar to Zif268, a "second wave" of IEG expression was not observed with c-Fos, as a significant increase in expression did not become apparent at eight hours. In contrast to Zif268, the expression of Arc and c-Fos appeared to be especially transient in medial prefrontal, retrosplenial and parietal cortices, which displayed very little immunoreactivity at four hours, suggesting their role in transcription and synaptic modification had been fully completed by this stage in these regions. Arc and c-Fos expression in the entorhinal and perirhinal cortices however appeared to show a similar trend to that of Zif268, continuing to decrease at eight hours. This suggests that synaptic downscaling may be a staggered process, involving the reduction in expression of different proteins at different times, and varying by region, which highlights the importance of using multiple markers of gene expression to fully characterise this process.

The findings of the current experiment are similar to the observations of Guzowski, Setlow [12], who found a peak of Arc, Zif268 and c-Fos mRNA expression in the hippocampus at $30 \mathrm{~min}$ following water maze training followed by a sharp drop at two hours, and returning to caged control levels or below at six hours, which approximately correspond to the $90 \mathrm{~min}$, four hour and eight hour time points for protein expression in this experiment. However neither set of results definitively rule out multiple waves of consolidation through IEG expression. Ramirez-Amaya, Vazdarjanova [10] observed a third wave of IEG expression at $24 \mathrm{~h}$ post-training, a time-point which was not assessed in this study. In fact there is evidence from other learning paradigms which point to a delayed expression of IEGs around this time. Katche, Goldin [24] found a delayed increase of Zif268 mRNA in the hippocampus at 12, 18 and $24 \mathrm{~h}$, but not nine or $30 \mathrm{~h}$ following inhibitory avoidance training. They subsequently used AS-ODNs to specifically knock down Zif268 expression in the hippocampus either during or after training. Zif268 AS-ODNs administered $30 \mathrm{~min}$ before training impaired memory measured both one and seven days following training, whereas AS-ODNs infused into the hippocampus eight hours following training spared memory at one day but not seven days. The authors postulate that the second wave of Zif268 expression observed between 12 and $24 \mathrm{~h}$ after training facilitated the persis- tence of long-term memory while not affecting its initial formation. Lonergan, Gafford [21] charted the time-course of Arc expression following fear conditioning, and found a moderate increase at $24 \mathrm{~h}$, although it did not reach statistical significance. Regardless, the possible existence of waves of IEG expression outside the timepoints assessed in this study do not explain the absence of increased Arc expression at eight hours. One possible explanation is the spatial learning in this study took place in familiar environment after three days of training as opposed to the novel environment used by Ramirez-Amaya, Vazdarjanova [10]. Therefore stimulus novelty may trigger multiple waves of consolidation, whereas repeated exposure to a familiar environment may encourage synaptic downscaling to prevent network hyper-excitability and allow for more subtle modifications of existing neural networks, an optimal scenario when learning incrementally about an environment across multiple days of training such as the water maze.

One possible factor which could influence the interpretation of these results is the absence of an additional control group who received training on the first two days of training, but not the third, in that the pre-learning IEG baseline level on day three could have been altered by previous training. However, given the nature of our findings, we feel any significant alterations in the IEG levels of this potential secondary control would not detract from our findings. If the pre-learning baseline were higher than our naïve controls, this would reveal an even more stark decrease below this baseline at eight hours which would emphasise the importance of our finding. On the other hand, a pre-learning baseline which was lower than the current naïve control, and not significantly different from our eight-hour time point would provide even more compelling evidence of a post-training reduction in neural activity, as this muted activity must have persisted from $24 \mathrm{~h}$ previously. This would also call into question the delayed wave of IEG expression observed at $24 \mathrm{~h}$ by Ramirez-Amaya, Vazdarjanova [10]. Therefore while the addition of such a control would be informative as to the origin and duration of the observed muted expression, it would not affect the conclusions of our experiment.

A further important consideration relevant to the multiple days of training employed in this experiment, and the use of Zif268 as a marker of neural activity is its demonstrated involvment in reconsolidation. Reconsolidation refers to the capacity for a memory trace to become transiently labile upon reactivation and vulnerable to interference during this time, a phenomenon which has been observed in a number of learning paradigms [25]. Blocking the expression of Zif268 in particular has been shown to disrupt previously consolidated contextual fear memories upon reactivation [26], and a similar effect has been observed in Zif68 KO mice in an object recognition task [27]. The memory disrupting effect of Zif268 AS-ODNs is also evident after consecutive days of contextual fear conditioning, suggesting that the role of Zif268 in reconsolidation is to strengthen and update existing memories [28]. Interestingly, however, while protein synthesis inhibition during a reactivation trial of a water maze task involving learning of a new spatial location every day disrupted initial memory for the previous days platform location, a similar impairment was not observed when the platform was kept constant for six days of training [29]. Therefore whether observed protein expression is a result of consolidation, reconsolidation or neural activity related to retrieval of a wellestablished memory trace may be dependent on either the number of learning trials or the passage of time since initial learning. Of relevance to the current study, the observed Zif268 expression on day three of training could well reflect reconsolidation processes as animals are still acquiring the task at this point and integrating new knowledge into their pre-existing memory representations [30]. The significance of the muted Zif268 expression observed here at eight hours post-training to reconsolidation processes is unclear, but may be enlightened by future studies looking at delayed Zif268 
expression following both short (one day) and long (six day) training protocols, conditions which are vulnerable and insensitive to disruption from reconsolidation respectively.

Another factor to consider with regards to the discrepancy between our findings and others, is the time of day of either training or sacrifice. IEG expression in rats is lowest during the day time, and higher at night time [31,32]. In the current experiment, all testing and sacrifice were carried out at evenly spaced intervals throughout the $12 \mathrm{~h}$ light cycle, and matched as closely as possible across all groups, to minimise the effects of circadian rythms on IEG expression. We assume that other groups have followed similar protocols therefore this is unlikely to explain the differences in findings.

Interestingly, despite the importance of the hippocampus for the acquisition of spatial memory [33], the brain regions in the current study which displayed unexpected muted activity at the eight hour time point, are generally thought to play more of a role in the long-term retrieval of spatial memory rather than its acquisition $[16,34,35]$. Therefore the activity patterns of the anterior cingulate, prelimbic and perirhinal cortices may be reflective of an early "synaptic tagging" process [36] which later facilitates the long-term integration of spatial memory into cortical networks over the course of systems consolidation.

An important caveat to bear in mind with regards to the current results is simply quantifying the overall number of active neurons may not be sufficiently sensitive to characterise the re-activation of a neural ensemble. The pattern of basal IEG expression during resting periods is altered by behavioural experience, in that the same neurons activated during training are the ones expressing IEGs at rest [8]. Therefore the activity of which neurons, rather than how many neurons, may be a more successful measure of IEG-facilitated consolidation, however the method employed in the current study was not designed to detect these differences. Furthermore, the order in which neurons replay events during resting periods may be equally important in the consolidation of spatial memory to the neocortex [37], which requires a level of temporal precision the IEG technique does not afford. However, neither of these factors can account for the reduction in neural activity below baseline at eight hours. The inclusion of Zif268 as a neural marker in the current study made it possible to detect such a decrease in activity, as its basal level of expression is quite high compared to other IEGs, where constitutive levels during resting periods are already low or absent [38], highlighting it as a useful and sensitive marker of reduced network activity.

\subsection{Conclusions}

This study did not find evidence for multiple waves of IEG expression following spatial learning, and reaffirm the initial couple of hours following learning as the critical time window for IEGfacilitated consolidation. However, the muted expression of IEG protein in the anterior cingulate, prelimbic and perirhinal cortices at eight hours following learning may be indicative of important neural changes in the late stages of cellular consolidation which pave the way for subsequent systems-level consolidation to the neocortex.

\section{Conflict of interests}

The authors declare no competing financial interests.

\section{Acknowledgements}

This work was funded in part by the Irish Research Council, and an NUIM John and Pat Hume scholarship held by D.N.B.

\section{References}

[1] J.L. McGaugh, Memory-a century of consolidation, Science 287 (5451) (2000) $248-251$.

[2] H.P. Davis, L.R. Squire, Protein synthesis and memory: a review, Psychol. Bull. 96 (3) (1984) 518-559.

[3] C. Katche, M. Cammarota, J.H. Medina, Molecular signatures and mechanisms of long-lasting memory consolidation and storage, Neurobiol. Learn. Mem. $106 C$ (2013) 40-47.

[4] D.F. Clayton, The genomic action potential, Neurobiol. Learn. Mem. 74 (3) (2000) 185-216.

[5] S. Renaudineau, et al., Impaired long-term stability of CA1 place cell representation in mice lacking the transcription factor zif268/egr1, Proc. Natl. Acad. Sci. U. S. A. 106 (28) (2009) 11771-11775.

[6] J. He, K. Yamada, T. Nabeshima, A role of Fos expression in the CA3 region of the hippocampus in spatial memory formation in rats, Neuropsychopharmacology 26 (2) (2002) 259-268.

[7] J.F. Guzowski, Insights into immediate-early gene function in hippocampal memory consolidation using antisense oligonucleotide and fluorescent imaging approaches, Hippocampus 12 (1) (2002) 86-104.

[8] D.F. Marrone, et al., Immediate-early gene expression at rest recapitulates recent experience, J. Neurosci. 28 (5) (2008) 1030-1033.

[9] S. Ribeiro, et al., Novel experience induces persistent sleep-dependent plasticity in the cortex but not in the hippocampus, Front. Neurosci. 1 (1) (2007) 43-55.

[10] V. Ramirez-Amaya, et al., Spatial exploration-induced Arc mRNA and protein expression: evidence for selective, network-specific reactivation, J. Neurosci. 25 (7) (2005) 1761-1768.

[11] Z. Penke, C. Chagneau, S. Laroche, Contribution of Egr1/zif268 to activity-dependent Arc/Arg3: 1 transcription in the dentate gyrus and area CA1 of the hippocampus, Front. Behav. Neurosci. 5 (2011) 48.

[12] J.F. Guzowski, et al., Experience-dependent gene expression in the rat hippocampus after spatial learning: a comparison of the immediate-early genes Arc, c-fos, and zif268, J. Neurosci. 21 (14) (2001) 5089-5098.

[13] R.G. Morris, Spatial localization does not require the presence of local cues, Learn. Motiv. 12 (2) (1981) 239-260.

[14] J. Kealy, et al., The effects of overtraining in the Morris water maze on allocentric and egocentric learning strategies in rats, Behav. Brain Res. 192 (2) (2008) 259-263.

[15] D.R. Harvey, et al., Emergence of an egocentric cue guiding and allocentric inferring strategy that mirrors hippocampal brain-derived neurotrophic factor (BDNF) expression in the Morris water maze, Neurobiol. Learn. Mem. 89 (4) (2008) 462-479.

[16] D.N. Barry, A.N. Coogan, S. Commins, The time course of systems consolidation of spatial memory from recent to remote retention: a comparison of the Immediate Early Genes Zif268, c-Fos and Arc, Neurobiol. Learn. Mem. 128 (2016) 46-55.

[17] M.M. Albasser, G.L. Poirier, J.P. Aggleton, Qualitatively different modes of perirhinal-hippocampal engagement when rats explore novel vs. familiar objects as revealed by c-Fos imaging, Eur. J. Neurosci. 31 (1) (2010) 134-147.

[18] S. Zangenehpour, A. Chaudhuri, Differential induction and decay curves of c-fos and zif268 revealed through dual activity maps, Brain Res. Mol. Brain Res. 109 (1-2) (2002) 221-225.

[19] L.A. Teather, et al., Differential induction of c-Jun and Fos-like proteins in rat hippocampus and dorsal striatum after training in two water maze tasks, Neurobiol. Learn. Mem. 84 (2) (2005) 75-84.

[20] K.L. Shires, J.P. Aggleton, Mapping immediate-early gene activity in the rat after place learning in a water-maze: the importance of matched control conditions, Eur. J. Neurosci. 28 (5) (2008) 982-996.

[21] M.E. Lonergan, et al., Time-dependent expression of Arc and zif268 after acquisition of fear conditioning, Neural Plast. 2010 (2010) 139891.

[22] N.C. O'Sullivan, et al., Temporal change in gene expression in the rat dentate gyrus following passive avoidance learning, J. Neurochem. 101 (4) (2007) 1085-1098.

[23] B. Siddoway, H. Hou, H. Xia, Molecular mechanisms of homeostatic synaptic downscaling, Neuropharmacology 78 (2014) 38-44.

[24] C. Katche, et al., Maintenance of long-term memory storage is dependent on late posttraining Egr-1 expression, Neurobiol. Learn. Mem. 98 (3) (2012) 220-227.

[25] K. Nader, O. Hardt, A single standard for memory: the case for reconsolidation, Nat. Rev. Neurosci. 10 (3) (2009) 224-234.

[26] J.L. Lee, B.J. Everitt, K.L. Thomas, Independent cellular processes for hippocampal memory consolidation and reconsolidation, Science 304 (5672) (2004) 839-843.

[27] B. Bozon, S. Davis, S. Laroche, A requirement for the immediate early gene zif268 in reconsolidation of recognition memory after retrieval, Neuron 40 (4) (2003) 695-701

[28] J.L. Lee, Memory reconsolidation mediates the strengthening of memories by additional learning, Nat. Neurosci. 11 (11) (2008) 1264-1266.

[29] R.G. Morris, et al., Memory reconsolidation: sensitivity of spatial memory to inhibition of protein synthesis in dorsal hippocampus during encoding and retrieval, Neuron 50 (3) (2006) 479-489.

[30] S. McKenzie, H. Eichenbaum, Consolidation and reconsolidation: two lives of memories? Neuron 71 (2) (2011) 224-233. 
[31] G. Grassi-Zucconi, et al., c-fos mRNA is spontaneously induced in the rat brain during the activity period of the circadian cycle, Eur. J. Neurosci. 5 (8) (1993) 1071-1078.

[32] C. Cirelli, G. Tononi, Differential expression of plasticity-related genes in waking and sleep and their regulation by the noradrenergic system, J. Neurosci. 20 (24) (2000) 9187-9194.

[33] R.G. Morris, et al., Place navigation impaired in rats with hippocampal lesions, Nature 297 (5868) (1982) 681-683.

[34] C.M. Teixeira, et al., Involvement of the anterior cingulate cortex in the expression of remote spatial memory, J. Neurosci. 26 (29) (2006) 7555-7564.
[35] J.M. Ramos, J.M. Vaquero, The perirhinal cortex of the rat is necessary for spatial memory retention long after but not soon after learning, Physiol. Behav. 86 (1-2) (2005) 118-127.

[36] E. Lesburgueres, et al., Early tagging of cortical networks is required for the formation of enduring associative memory, Science 331 (6019) (2011) 924-928.

[37] H.F. Olafsdottir, F. Carpenter, C. Barry, Coordinated grid and place cell replay during rest, Nat. Neurosci. 19 (6) (2016) 792-794.

[38] L. Kaczmarek, A. Chaudhuri, Sensory regulation of immediate-early gene expression in mammalian visual cortex: implications for functional mapping and neural plasticity, Brain Res. Brain Res. Rev. 23 (3) (1997) 237-256. 